\title{
Ultrastructural Characteristics of Sheep and Horse Mesenchymal Stem Cells (MSCs)
}

\author{
Asuman Ozen ${ }^{1}$, Irem Gul Sancak ${ }^{2 *}$, Brigitte Von Rechenberg ${ }^{3}$, Sabine Koch $^{3}$ \\ ${ }^{1}$ Department of Histology-Embryology, Veterinary Faculty, Ankara University, Ankara, Turkey; ${ }^{2}$ Department of Surgery, Veterinary \\ Faculty, Ankara University, Ankara, Turkey; ${ }^{3}$ Competence Center for Applied Biotechnology and Molecular Medicine (CABMM), \\ Vetsuisse Faculty, University of Zurich, Zurich, Switzerland. \\ Email: *iremgulsancak@gmail.com
}

Received July $15^{\text {th }}, 2013$; revised August $28^{\text {th }}, 2013$; accepted September $29^{\text {th }}, 2013$

Copyright (C) 2013 Asuman Ozen et al. This is an open access article distributed under the Creative Commons Attribution License, which permits unrestricted use, distribution, and reproduction in any medium, provided the original work is properly cited.

\begin{abstract}
In the present study, the morphological and ultrastructural characteristics of mesenchymal stem cells (MSCs) induced towards osteogenic, adipogenic, and chondrogenic lineages were investigated. The main objective of this paper was to focus on the differentiation capacity of ovine [oMSCs] and equine MSCs [eMSCs]. Bone marrow [BM] MSCs were isolated from ovine and equine patients, expanded in monolayer culture and induced into osteogenic, adipogenic and chondrogenic differentiation. For chondrogenic differentiation, cells were cultured in micromass culture. Also, their ultrastructural phenotypes were studied by transmission electron microscopy [TEM]. This study was aimed to provide an indepth morphological description of BM-derived ovine and equine MSCs induced towards three lineages, it demonstrated that TEM analysis is useful in elucidating detailed structural information.
\end{abstract}

Keywords: Ultrastructure; Mesenchymal Stem Cells (MSCs); TEM; Differentiation; Equine; Ovine

\section{Introduction}

Mesenchymal stem cells (MSCs) are self-renewing and multipotent cells, which have the potential to differentiate into various tissues as bone, cartilage, fat, muscle and tendon under appropriate conditions. In view of this capacity, MSCs represent a promising model for tissue engineering and regenerative medicine. First isolated from bone marrow [1], MSCs typically represent a fibroblastlike cell population [2]. They have the ability to differentiate into multiple mesenchymal cell types such as adipocytes, osteoblasts and chondroblasts [3]. During differentiation phenotypical changes occur, which trigger protein synthesis in the cell. It has been reported that during the differentiation process collagene and elastin synthesis and the number of intracellular organelles and electrodense granules increase [4]. Moreover, MSCs have immunomodulatory and anti-inflammatory functions, which make them good candidates for the treatment of diseases with cell therapy. Although MSCs have been well studied, their ultrastructure has not been fully investigated and detailed.

Animals are widely used as a model for humans. In

${ }^{*}$ Corresponding author. human medicine, new therapies are tested in animals before they are used for the treatment of diseases. Therefore, the cell characteristics, phenotype and surface markers of animal MSCs are important for future research in this field. Despite intensive research having been conducted on MSCs, there is no broadly accepted definitive phenotype or surface marker for ovine or equine cells. Thus there is a need for the morphological and surface marker analysis of these cells. Therefore, the aim of this study was to evaluate the ultrastructure of ovine and equine MSCs and to define their surface markers in order to highlight the morphological changes occurring in these cells after they are stimulated into trilineage differentiation.

\section{Materials and Methods}

\subsection{Isolation and Culturing of MSCs}

Blood samples were taken from three horses at a mean age of 10 years and from three sheep at a mean age of 2 years prior to their euthanasia. Sampling was performed in compliance with animal protection guidelines. Heparinized bone marrow aspirates were collected from the horses $(35 \mathrm{ml})$ and sheep $(20 \mathrm{ml})$ under general anesthe- 
sia. Mononuclear cells were isolated by gradient density centrifugation and resuspended in DMEM-LG (low glucose) $(1 \mathrm{~g} / \mathrm{l})$ containing $1 \%$ pen/strep and $10 \%$ fetal bovine serum (FBS, Gibco/Invitrogen AG, Life Technologies, Basel, Switzerland). The cells were incubated at $37^{\circ} \mathrm{C}$ in a humidified atmosphere containing $5 \% \mathrm{CO}_{2}$. The medium was changed every 3 days. On days 12 to 14 , cells were detached using $0.25 \%$ trypsin-EDTA (Gibco/Invitrogen AG, Life Technologies, Basel, Switzerland) and replated ( $1^{\text {st }}$ passage $)$.

\subsection{Differentiation Protocol}

In order to characterize harvested cells, passage three cells were stimulated into adipogenic and osteogenic differentiation in monolayer culture. For chondrogenesis, a micromass pellet was used $\left(5.0 \times 10^{5}\right.$ cells $\left./ \mathrm{cm}^{2}\right)$ [5].

For osteogenic differentiation the cells were placed in a six-well plate at a density of $5.0 \times 10^{4}$ cells $/ \mathrm{cm}^{2}$. An osteogenic induction medium consisting of DMEM- LG, $0.05 \mathrm{mM}$ ascorbate-2-phosphate, $100 \mathrm{nM}$ dexametasone, and $10 \mathrm{mM}$ sodium $\beta$-glycerophosphate (Sigma-Aldrich, Buchs, Switzerland). The cells were cultured in monolayer culture for a period of three weeks and the medium was changed twice a week [6].

For adipogenic differentiation, the cells were seeded at the same density. In total the cells were stimulated for 18 days. The cells were first exposed to a lipogenic induction medium consisting of standard medium high glucose DMEM (10\% FBS), 0.5 mM 3-isobutyl-1-methylxantine, $1 \mu \mathrm{M}$ dexamethasone, $10 \mu \mathrm{g} / \mathrm{ml}$ insulin, $0.5 \mathrm{mM}$ indomethacin (Sigma-Aldrich, Buchs, Switzerland) for 72 hours. Later, the cells were exposed to an adipogenic maintenance medium for 2 days. This treatment cycle was repeated twice [5].

For chondrogenic differentiation, pellets were stimulated for 18 days in standard medium high-glucose DMEM containing $6.25 \mu \mathrm{g} / \mathrm{ml}$ insulin-transferrin-selenious acid, $0.1 \mathrm{mM}$ ascorbate-2-phosphate, $10^{7} \mathrm{M}$ dexamethasone, $1.25 \mathrm{mg} / \mathrm{ml}$ bovine serum albumin, 5000 $\mathrm{IU} / \mathrm{ml}$ penicillin, $5000 \mu \mathrm{g} / \mathrm{ml}$ streptomycin, $50 \mu \mathrm{g} / \mathrm{ml}$ ascorbate 2-phosphate and $100 \mathrm{nM}$ dexamethasone and $10 \mathrm{ng} / \mathrm{ml}$ human transforming growth factor [6].

\subsection{Histochemical Staining}

The differentiation of cells into the adipogenic, osteogenic, and chondrogenic lineages was determined using different histological staining techniques. Von Kossa staining demonstrated deposition of minerals in osteogenic cultures. Adipogenic differentiation was evaluated using Oil red $\mathrm{O}$ to reveal lipid droplet accumulation in the cell cytoplasm and Alcian blue ( $\mathrm{pH}$ 2.5) staining was used to reveal chondrogenic differentiation based on the production of a ground substance matrix. In all these staining methods, the nuclei were counterstained with Mayer's hematoxylin and the stained cells were examined under a light microscope.

\subsection{Transmission Electron Microscopy (TEM)}

In order to evaluate the differentiation process of ovine and equine MSCs, into three lineages, the cells were fixed before (Day 0) and after differentiation (day 21 in chondrogenic and osteogenic differentiation and day 18 in adipogenic differentiation). On day 0 the cells were grown and fixed in the flasks, but the cells seeded for three lineage differentiation were kept in the 12-well plates and following differentiation were fixed in the wells using $0.8 \%$ formaldehyde and $1.25 \%$ gluteraldehyde in $100 \mathrm{mM}$ phosphate buffer solution at $37^{\circ} \mathrm{C} 2-16$ hours. Subsequently, the cells were washed with $0.5 \%$ BSA in PBS. For removal, a cell scraper was used and debris was centrifuged at $3000 \mathrm{~g}$ for 3 minutes. The pellet was mixed with $2 \%$ agar, centrifuged at $4000 \mathrm{~g}$ for 2 minutes and post fixed in $1 \%$ osmium tetroxide. The samples were dehydrated in graded ethanol, passed through propylene oxide and embedded in epon-araldite. The sections were examined in TEM (Zeiss EM 912 Omega $\mathrm{AB}$ ) and photographed with a Gatan Orius SC $200 \mathrm{~W}$ CCD camera.

\section{Results}

\subsection{Isolation and Cell Culture}

The Third passage ovine and equine MSCs were grown in T25 culture flasks and showed fibroblast-like morphology. At the beginning of the culture period, ovine cells showed better proliferation than equine MSCs. But overall, throughout the culture period, the confluence of the cells was similar (12 - 15 days). While the ovine MSCs were homogeneously distributed, the equine cells formed colonies in the flask. By the end of the second week, both the ovine and equine MSCs displayed interconnection and monolayer confluence was observed.

\subsection{Light Microscopy and Staining}

The osteogenic induction of MSCs resulted in calcium deposition in the colonies, which could be visualized with Von Kossa staining, while the adipogenic induction of MSCs resulted in lipid droplet accumulation in the cells, which could be visualized with Oil red O staining. The oil droplets were clearly visible on day 18 post-differentiation in both ovine and equine cells. Chondrogenic induction was investigated using high-density cell pellet cultures in conditioned media to form 3D cell spheres with the help of TGF- $\beta 1$. After 3 weeks, cell spheres consisted of a cell population with a heterogeneous extracellular matrix. Alcian blue staining of the differenti- 
ated 3D cell pellets confirmed the production of glycosaminoglycans and glycoproteins.

\subsection{Transmission Electron Microscopy (TEM)}

Positioning the presence of a euchromatic nucleus associated with abundant mitochondria, Golgi complexes and rough endoplasmic reticula, briefly, cell organelles being high in number is considered an indicator of an active cell. At the beginning of differentiation, no difference was noted between ovine and equine cells (Figure 1). Pseudopodia-like structures were observed on each side of the cells and it was considered that these structures may have a role in signalization (Figure 2).

Adipogenic differentiation was characterized by abundant mitochondria and lipid droplets and multiple smooth endoplasmic reticula (Figure 3).

In both equine and ovine samples, osteogenic differentiation was characterized by an increased number of autophagosomes. The abundance of autophagosomes could be an indicator of the medium being inappropriate. During the process of osteogenic differentiation, the presence of granular endoplasmic reticula, ribosomes, Golgi complexes, mitochondria and an euchromatic nucleus was observed (Figure 4).

Transverse and longitudinal sections pertaining to the chondrogenic lineage presented with vacuoles in the extracellular space and abundant collagen. During chondrogenic differentiation, the inner cells of the micromass culture resembled normal cartilaginous cells, while the cultured cell spheres were surrounded by a thin capsule consisting of long, elongated, fibroblast-like cells. In these cells, granular endoplasmic reticula and free ribosomes and polisomes were abundant (Figure 5).
The most significant finding, both at the beginning of differentiation and in the differentiated cells, was the continuous contact of mesenchymal cells with each other. At the contact sites of the cell membrane, an increased number of vacuoles was observed (Figure 6), which is thought to play a significant role in the signaling of mesenchymal cells.

\section{Discussion}

MSCs have been studied extensively for their ability to self-renew and to differentiate into cell types such as osteoblasts, chondrocytes and adipocytes $[2,3,7,8]$. This study, for the first time, examined the differentiation pathway of ovine and equine mesenchymal stem cells in detail using a transmission electron microscope.

As described in previous studies [9,10], these cells typically contain 2 different zones ultrastructurally. While the organelles are localized around the nucleus, the organelles are not visible in the peripheral part. In the present study, ovine and equine MSCs displayed similar characteristics.

The osteogenic induction of MSCs resulted in calcium deposition in the colonies, which could be visualized using Von Kossa staining. The ultrastructural analysis of ovine and equine differentiated cell types confirmed the acquisition of an osteoblastic phenotype. In the cytoplasm of both ovine and equine cells, the accumulation of multiple matrix vesicles was seen. MSCs represent a more immature type of in vitro osteogenesis. This is thought to be due to the microenvironmental predisposition of the MSCs. They tend to grow better in 3-D cultures [6].

The adipogenic differentiation of MSCs resulted in

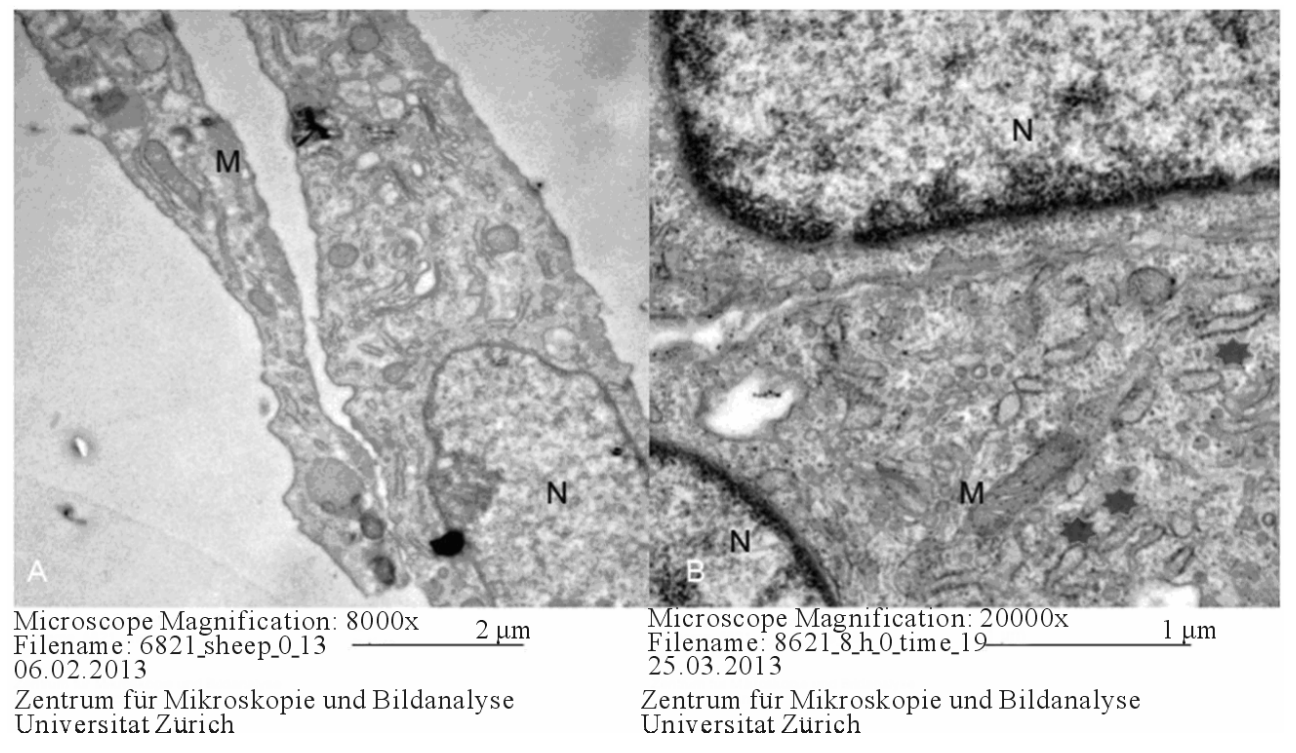

Figure 1. Ovine MSC (A) and Equine MSCs (B) on day 0. N: nucleus, M: mitochondria, Asterix: rough endoplasmic reticulum. 
positive Oil red O staining, which proved that lipid droplet accumulation was achieved in monolayer cultures. These lipid droplets were clearly visible at ultrastructural level. Adipogenesis was achieved with the induction of MSCs by the cell cycling method, in which the stimulated cells were initially cultured in an adipogenesisinducing medium and for another 3 days in adipogenesismaintenance medium. This stimulation was repeated twice, and the treatment was continued for 18 days in total. This cell cycling method was found to be useful in adipogenic differentiation and enabled multiple welldefined lipid droplets to be seen by both light and transmission electron microscopy (TEM).

In vitro cartilage formation by MSCs is observed when cells are cultured as a micromass in a defined medium for chondrogenesis [11]. Cells located in the center of the micromass showed many characteristics of metabolically active cells. Their cytoplasm contained numerous matrix vesicles. Also their shape differed from the MSCs localized in the peripheral zone. Central cells had the typical appearance of mature chondrocytes, they were round and rich in matrix. In addition, large amounts of proteoglycans and collagens filled the intercellular space between the cells.

Pasquinelli et al. [12] have compared the fine structure of bone marrow-derived human mesenchymal cells with that of amnion and chorion-derived mesenchymal. These researchers reported that, in human mesenchymal cells originating from the bone marrow, the cisternae of the rER were enlarged and multifocal electron light vesicles were concentrated in the periphery. The peripheral ac- cumulation of the vesicles was attributed to the niche microenvironment. Mesenchymal cells of chorionic origin were reported to have a simpler fine structure and lower metabolic rate. It has been indicated that, in these cells, clusters of rER and glycogen accumulation were also located peripherally. Furthermore, the presence of superficial microvilli and intercellular connections has been reported in mesenchymal cells originating from the amniotic membrane. It has been ascertained that mesen-

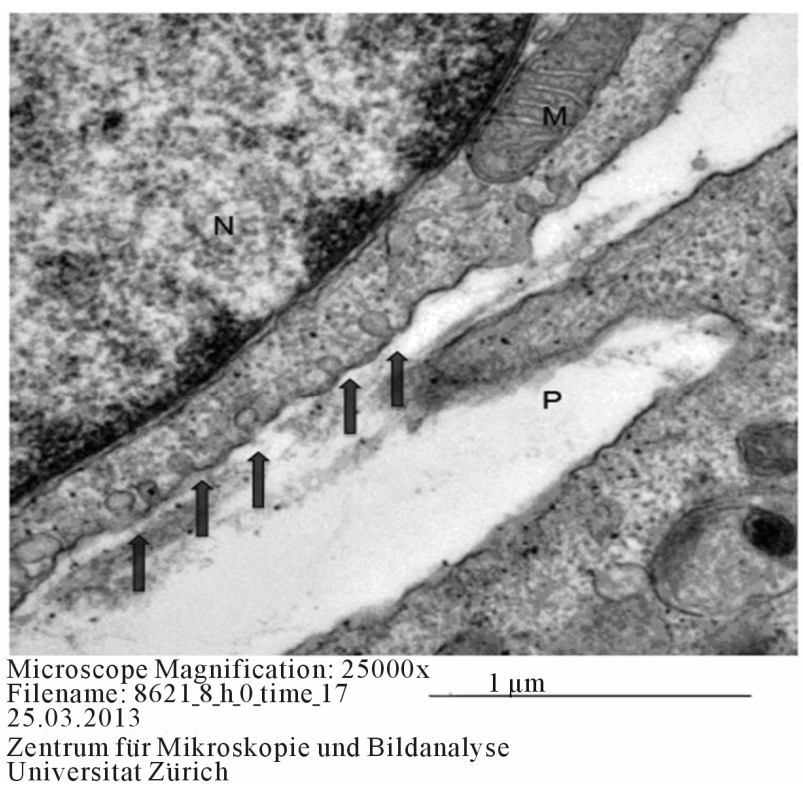

Figure 2. Pseudopod-like structures (P), Arrows: Vacuoles, $\mathrm{N}$ : nucleus, M: mitochondria.

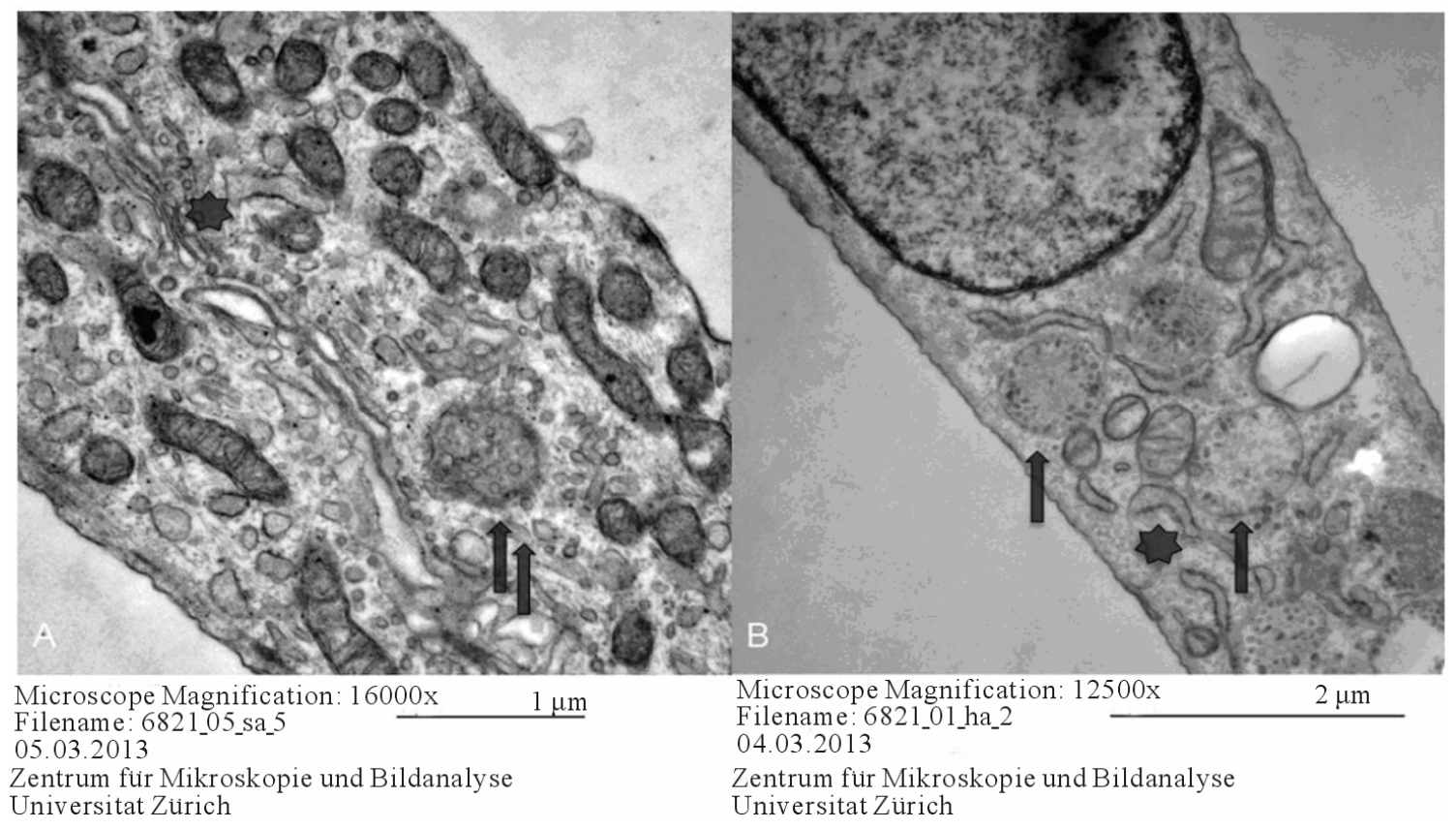

Figure 3. Adipogenic differentiation in ovine (A) and equine (B) MSCs. M: mitochondria, Arrows: lipid droplets, Asterix: smooth endoplasmic reticulum. 


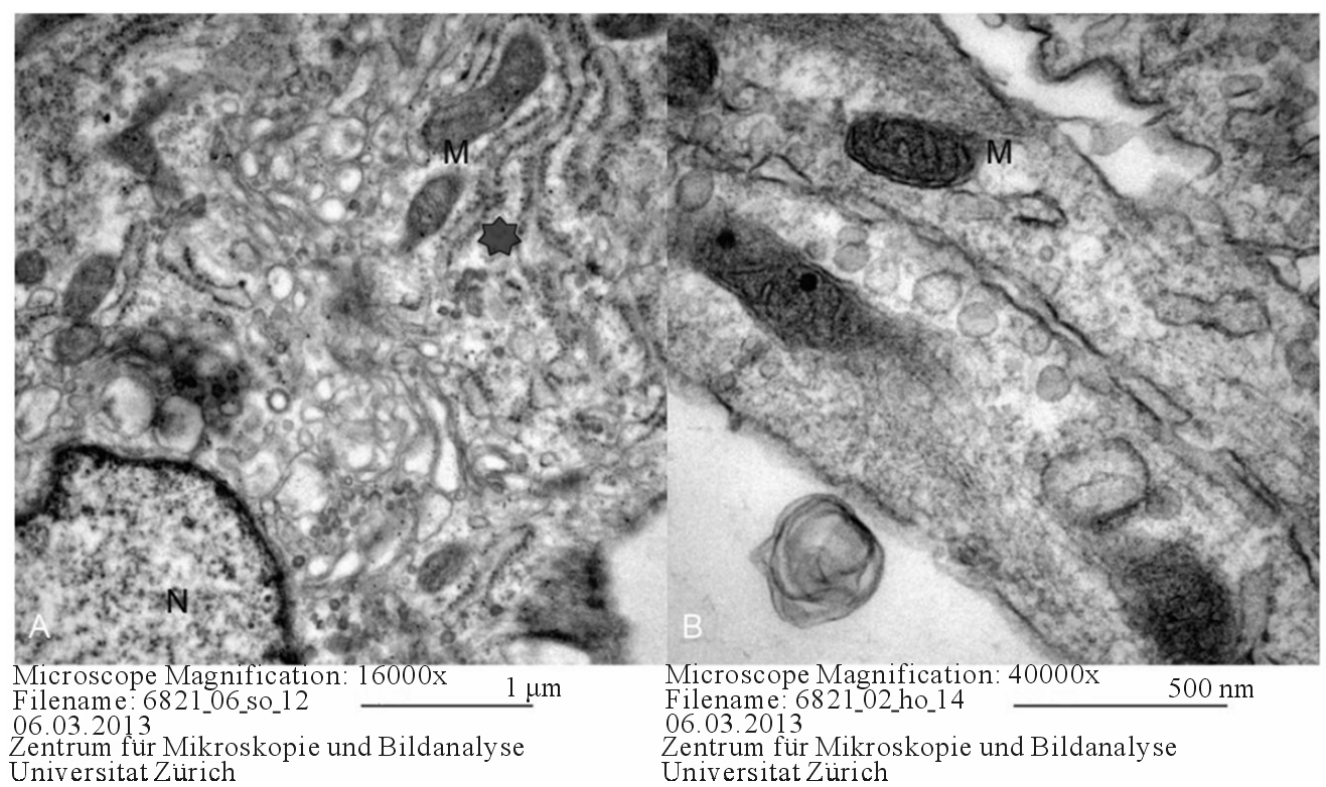

Figure 4. Osteogenic differentiation in ovine (A) and equine (B) MSCs. M: mitochondria, N: nucleus, Asterix: rough endoplasmic reticulum.

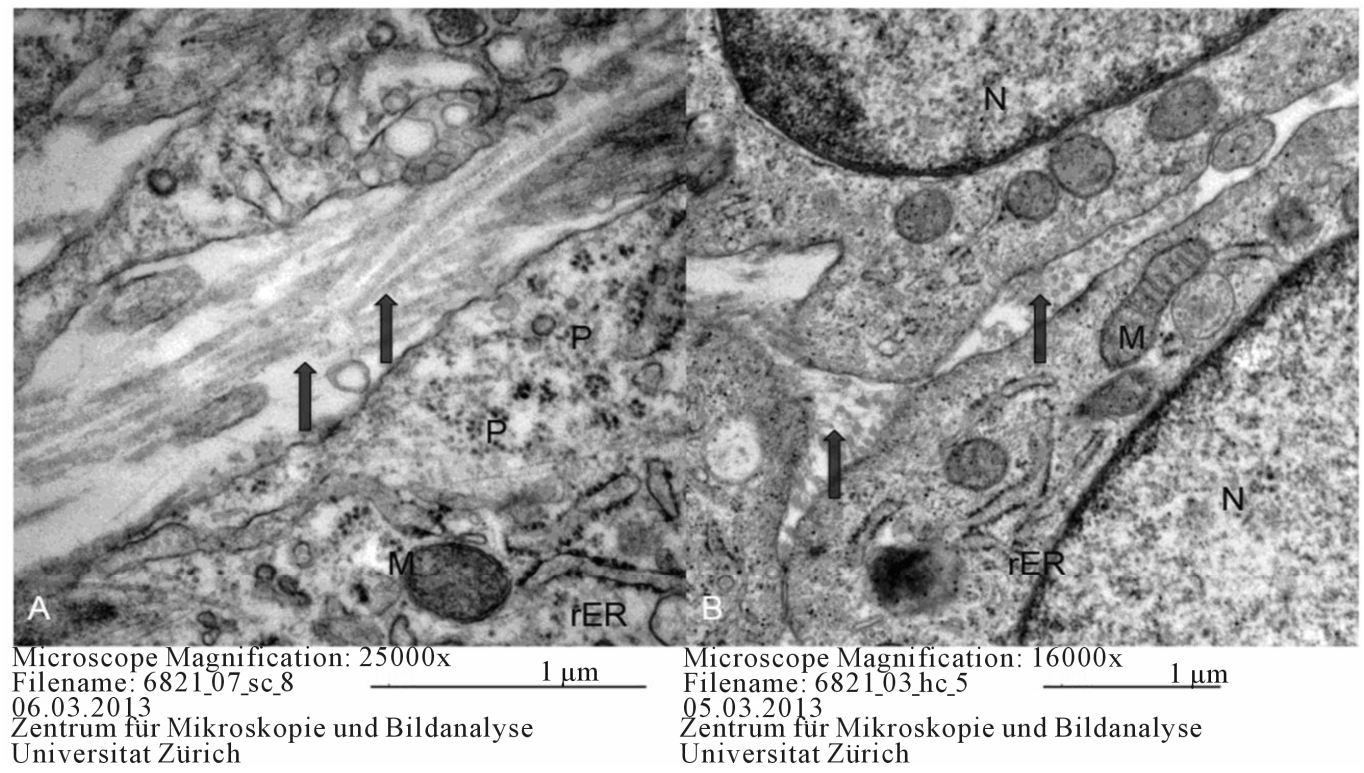

Figure 5. Chondrogenic differentiation in ovine (A) and equine (B) MSCs. Arrows: collagen, M: mitochondria, N: Nucleus, rER: rough endoplasmic reticulum, $P$ : polisome.

chymal cells of amniotic membrane display features suggesting them to have pluripotent properties. In the present study, the fine structure of equine and ovine bone marrow-derived stem cells was investigated, and it was determined that their ultrastructure displayed similarity to that of human bone marrow-derived mesenchymal cells. Equine and ovine mesenchymal cells of bone marrow origin were observed to contain an euchromatic nucleus, rER cisternae and abundant mitochondria. In the present study, the vacuoles, which opened into the exterior of the cell membrane, having increased particularly in the cell-cell contact sites, was in agreement with the observation of the peripheral accumulation of electron light vesicles in previously conducted research. The concentration of an increased number of vacuoles in the periphery and these vacuoles opening into the cell surface were both considered to be related to the involvement of these vacuoles in signaling. In a ccanning electron microscopical study, Teti et al. [13] examined the vacuoles found beneath the cell membrane and determined them to be related with increased protein synthesis. In the present study, these vacuoles were observed even 


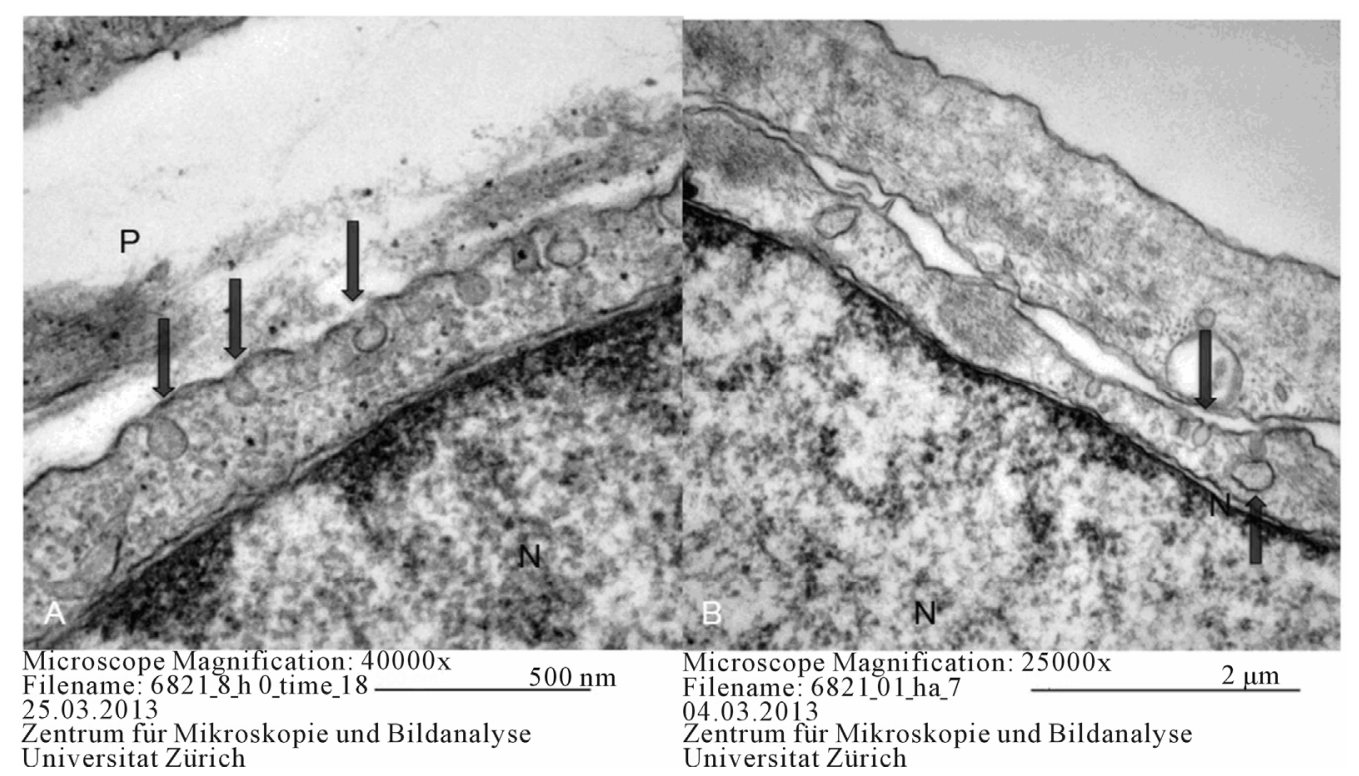

Figure 6. Contact areas in equine MSCs on day 0 (A) and in equine adipogenic differentiation (B) P: pseudopod-like structure, N: nucleus, Arrows: vacuoles.

in undifferentiated cells and they were considered to be most likely related to signalization in cells.

In a study on canine mesenchymal cells, Csaki et al. [14] investigated the ultrastructure of chondrogenic, osteogenic and adipogenic differentiation. These researchers reported that, in osteogenic differentiation, a large amount of fibrils, mainly composed of collagen type I fibrils, existed in the extracellular area. In adipogenic differentiation, a very large number of lipid droplets, well-developed rER and an excessive number of large mitochondria with abundant lamellar cristae were observed. On the other hand, in chondrogenic differentiation, round cells that resembled chondrocytes were located in the inner part, and fibrocyte-like squamous cells were located in the outer part. The presence of proteoglycan chains was determined in the extracellular matrix. The findings obtained in the present study were in agreement with those reported by the researchers referred to above, and in chondrogenic differentiation, transversal and longitudinal collagen sections were observed inbetween the cells.

Karaöz et al. [9] have reported multiple differences between rat mesenchymal cells obtained from the bone marrow and mesenchymal stem cells isolated from pancreatic islets, in terms of ultrastructure. These researchers observed that the number of lipid droplets in stem cells isolated from the pancreas was lower than that of the mesenchymal cells obtained from the bone marrow. Enlarged ER cisternae, which were filled with electron dense material, were observed in both cell groups. However, these cisternae had a more oval shape in mesenchymal cells isolated from the pancreatic islets, while those found in the bone marrow-derived mesenchymal cells appeared as enlarged cisternae. While mesenchymal cells obtained from the bone marrow were covered with microvilli, those isolated from the pancreatic islets had thick extensions that resembled pseudopods. Similarly, in the present study, it was observed that the cells communicated with each other by means of pseudopod-like structures. At these cell-cell contact sites, interconnections were observed to exist between the cells.

Raimondo et al. [10] described the pseudopod-like structures found on the cell membrane. We have also observed these structures as previously described. Pseudopod-like structures are more likely related to cell signalization and their numbers are abundant at the beginning of differentiation. When the cells reach a certain confluence, the number of the vacuoles and pseudopodia decreases. The perinuclear localization of heterochromatin in the nucleus has been described. Similar to the findings obtained in the present study, both rounded and elongated profiles and thick cristae were observed in the mitochondria and typical vesicles and vacuoles were shown in the golgi complexes.

This study was aimed to provide an in-depth morphological description of both ovine and equine bone-marrow derived MSCs induced towards adipogenic, osteogenic, and chondrogenic lineages, and it was demonstrated that electron microscopical analysis is useful in elucidating detailed structural information. Moreover, the demonstration of ultrastructural changes provides indisputable evidence of differentiation. It is considered that the ultrastructural investigation of the fine structure of mesenchymal cells and their osteogenic, chondrogenic and adipogenic differentiation would contribute to regenerative medicine and tissue engineering through the 
provision of alternative material from connective tissue. Therefore, further studies are required to be conducted in this field.

\section{REFERENCES}

[1] A. J. Friedenstein, J. F. Gorskaja and N. N. Kulagina, "Fibroblast Precursors in Normal and Irradiated Mouse Hematopoietic Organs," Experimental Hematology, Vol. 4, No. 5, 1976, pp. 267-274.

[2] D. J. Prockop, "Marrow Stromal Cells as Stem Cells for Nonhematopoietic Tissues," Science, Vol. 4, No. 276, 1997, pp. 71-74. http://dx.doi.org/10.1126/science.276.5309.71

[3] M. F. Pittenger, A. M. Mackay, S. C. Beck, R. K. Jaiswal, R. Douglas, J. D. Mosca, M. A. Moorman, D. W. Simonetti, S. Craig and D. R. Marshak, "Multilineage Potential of Adult Human Mesenchymal Stem Cells," Science, Vol. 284, No. 5411, 1999, pp. 143-147. http://dx.doi.org/10.1126/science.284.5411.143

[4] C. E. Sarraf, W. R. Otto and M. Eastwood, "In Vitro Mesenchymal Stem Cell Differentiation after Mechanical Stimulation," Cell Proliferation, Vol. 44, No. 1, 2011, pp. 99-108. http://dx.doi.org/10.1111/j.1365-2184.2010.00740.x

[5] V. Lettry, K. Hosoya, S. Takagi and M. Okumura, "Coculture of Equine Mesenchymal Stem Cells and Mature Equine Articular Chondrocytes Results in Improved Chondrogenic Differentiation of the Stem Cells," Japanese Journal of Veterinary Research, Vol. 58, No. 1, 2010, pp. 5-15.

[6] J. Koerner, D. Nesic, J. D. Romero, W. Brehm, P. Mainil-Varlet and S. P. Grogan, "Equine Peripheral BloodDerived Progenitors in Comparison to Bone Marrow- Derived Mesenchymal Stem Cells," Stem Cells, Vol. 24, No. 6, 2006, pp. 1613-1619.

http://dx.doi.org/10.1634/stemcells.2005-0264

[7] F. Barry and J. Murphy, "Mesenchymal Stem Cells: Clinical Applications and Biological Characterization," The International Journal of Biochemistry \& Cell Biology, Vol. 36, No. 4, 2004, pp. 568-584.

[8] H. Castro-Malaspina, R. E. Gay, G. Resnick, N. Kapoor, P. Meyers, D. Chiarieri, S. McKenzie, H. E. Broxmeyer and M. A. Moore, "Characterization of Human Bone Marrow Fibroblast Colony-Forming Cells (CFU-F) and Their Progeny," Blood, Vol. 56, No. 2, 1980, pp. 289301.

[9] E. Karaoz, A. Aksoy, S. Ayhan, A. E. Sariboyaci, F. Kaymaz and M. Kasap, "Characterization of Mesenchymal Stem Cells from Rat Bone Marrow: Ultrastructural Properties, Differentiation Potential and Immunophenotypic Markers," Histochemistry and Cell Biology, Vol. 132, No. 5, 2009, pp. 533-546. http://dx.doi.org/10.1007/s00418-009-0629-6

[10] S. Raimondo, C. Penna, P. Pagliaro and S. Geuna, "Morphological Characterization of GFP Stably Transfected Adult Mesenchymal Bone Marrow Stem Cells," Journal of Anatomy, Vol. 208, No. 1, 2006, pp. 3-12. http://dx.doi.org/10.1111/j.1469-7580.2006.00511.x

[11] I. Sekiya, D. C. Colter and D. J. Prockop, "BMP-6 Enhances Chondrogenesis in a Subpopulation of Human Marrow Stromal Cells," Biochemical and Biophysical Research Communications, Vol. 284, No. 2, 2001, pp. 411-418. http://dx.doi.org/10.1006/bbrc.2001.4898

[12] G. Pasquinelli, P. Tazzari, F. Ricci, C. Vaselli, M. Buzzi, R. Conte, C. Orrico, L. Foroni, A. Stella, F. Alviano, G. P. Bagnara and E. Lucarelli, "Ultrastructural Characteristics of Human Mesenchymal Stromal (Stem) Cells Derived From Bone Marrow and Term Placenta," Ultrastructural Pathology, Vol. 31, No. 1, 2007, pp. 23-31. http://dx.doi.org/10.1080/01913120601169477

[13] G. Teti, C. Cavallo, B. Grigolo, S. Giannini, A. Facchini, A. Mazzotti and M. Falconi, "Ultrastructural Analysis of Human Bone Marrow Mesenchymal Stem Cells during in Vitro Osteogenesis and Chondrogenesis," Microscopy Research and Technique, Vol. 75, No. 5, 2012, pp. 596-604. http://dx.doi.org/10.1002/jemt.21096

[14] C. Csaki, U. Matis, A. Mobasheri, H. Ye and M. Shakibaei, "Chondrogenesis, Osteogenesis and Adipogenesis of Canine Mesenchymal Stem Cells: A Biochemical, Morphological and Ultrastructural Study," Histochemistry and Cell Biology, Vol. 128, No. 6, 2007, pp. 507-520. http://dx.doi.org/10.1007/s00418-007-0337-z 